\title{
Establishing a cost-effective national surveillance system for Bluetongue using scenario tree modelling
}

\author{
Daniela C. Hadorn ${ }^{1 *}$, Vanessa Racloz ${ }^{1,2}$, Heinzpeter SCHWERmer ${ }^{1}$, \\ Katharina D.C. STÄRK ${ }^{1,3}$ \\ ${ }^{1}$ Federal Veterinary Office, Schwarzenburgstrasse 155, CH-3003 Bern, Switzerland \\ ${ }^{2}$ Swiss Tropical Institute, Basel, Switzerland \\ ${ }^{3}$ Royal Veterinary College, London, United Kingdom
}

(Received 19 January 2009; accepted 13 July 2009)

\begin{abstract}
Vector-borne diseases pose a special challenge to veterinary authorities due to complex and time-consuming surveillance programs taking into account vector habitat. Using stochastic scenario tree modelling, each possible surveillance activity of a future surveillance system can be evaluated with regard to its sensitivity and the expected cost. The overall sensitivity of various potential surveillance systems, composed of different combinations of surveillance activities, is calculated and the proposed surveillance system is optimized with respect to the considered surveillance activities, the sensitivity and the cost. The objective of this project was to use stochastic scenario tree modelling in combination with a simple cost analysis in order to develop the national surveillance system for Bluetongue in Switzerland. This surveillance system was established due to the emerging outbreak of Bluetongue virus serotype 8 (BTV-8) in Northern Europe in 2006. Based on the modelling results, it was decided to implement an improved passive clinical surveillance in cattle and sheep through campaigns in order to increase disease awareness alongside a targeted bulk milk testing strategy in 200 dairy cattle herds located in high-risk areas. The estimated median probability of detection of cases (i.e. sensitivity) of the surveillance system in this combined approach was $96.4 \%$. The evaluation of the prospective national surveillance system predicted that passive clinical surveillance in cattle would provide the highest probability to detect BTV8 infected animals, followed by passive clinical surveillance in sheep and bulk milk testing of 200 dairy cattle farms in high-risk areas. This approach is also applicable in other countries and to other epidemic diseases.
\end{abstract}

national surveillance system / cost-effective / scenario tree modelling / Bluetongue / Switzerland

\section{INTRODUCTION}

Bluetongue (BT) is a vector-borne viral disease, which affects domestic and wild ruminants. Infected animals can display a variety of clinical symptoms such as fever, apathy, anorexia, nasal discharges, lameness or abortions.

\footnotetext{
* Corresponding author: Daniela.Hadorn@bvet. admin.ch
}

The virus, of which there are currently 24 serotypes known, is a member of the Reoviridae family and Orbivirus genus. BT is exclusively related to the presence of the virus in the competent vector, i.e. insects of the Culicoides species, and is spread by different species of Culicoides midges depending on the geographic area and climatic factors [7]. In Europe, BT virus (serotypes 1, 2, 4, 9, and 16) has been circulating on a regular basis only in the

This is an Open Access article distributed under the terms of the Creative Commons Attribution-Noncommercial License (http://creativecommons.org/licenses/by-nc/3.0/), which permits unrestricted use, distribution, and reproduction in any noncommercial medium, provided the original work is properly cited. 
Mediterranean and Balkan regions since the late $1990 s^{1}$. Before, sporadic outbreaks occurred in the 1950s in Portugal and Spain, causing great economic losses in the sheep industry. Therefore, the detection of Bluetongue virus serotype 8 (BTV-8) in northern parts of Europe in August 2006 was unexpected. It was first reported in the Netherlands and Belgium, then in Germany, Luxembourg, and France $[5,6]$. Although no further cases were detected during the winter period of 2006-2007, BTV-8 was rediscovered in sentinel cattle in spring of 2007 in Germany [5]. Due to its rapid spread southwards towards Switzerland, Swiss policy makers decided to establish a national surveillance system for BTV-8 starting in spring 2007 with the aim of detecting the first BTV-8 cases as early as possible.

The World Animal Health Organization $(\mathrm{OIE})^{2}$ defines a surveillance system as a method of surveillance that may involve one or more component activities that generates information on the health, disease or zoonosis status of animal populations. The component activities of such a surveillance system may be active or passive $[1,3]$. A surveillance system component (SSC) consists of a logical sequence of states and actions linking the root node of the scenario tree with different end notes of this tree. Nodes within the tree are linked with weighted edges. The edge weights are generally given by probabilities [4]. An advantage of this tool is its ability to take into account infection risks and probabilities of detection.

With regard to infection risks and probabilities of detection, the population can be stratified into different risk strata. Within each stratum, all units (e.g. farms) have the same probability of becoming infected and subsequently detected given the population is infected at or above a certain prevalence, i.e. the design prevalence

\footnotetext{
${ }^{1}$ Purse B.V., Baylis M., McCormick B.J.J., Rogers D., Foresight project "Infectious diseases: preparing for the future" T.8.3: Bluetongue in Europe, Department of Trade and Industry, 2006.

2 OIE, Terrestrial Animal Health Code of the OIE [on line] (2008) http://www.oie.int/eng/normes/ mcode/en_chapitre_1.1.4.htm [consulted 20 October 2008].
}

[4]. The basis of targeted surveillance is to stratify the population according to risk and then sample units more intensively from high-risk strata [2].

In order to design a successful and costeffective early warning system with regard to BTV-8 in Switzerland, stochastic scenario tree modelling was used [2, 4]. This enabled the evaluation of different SSC with regard to their sensitivities and financial implications, all within the Swiss context in terms of geography, resources and infrastructure. The sensitivity of a SSC is the probability that the SSC will give a positive outcome and the disease will become detected [4].

The objective of this study was to apply scenario tree modelling in order to develop an optimal cost-effective national surveillance system for emerging vector-borne diseases and to analyze the effectiveness of the prospective system. The approach was developed on the example of the national surveillance system for BTV-8 in Switzerland. But this approach is also applicable in other countries and to other epidemic diseases.

\section{MATERIALS AND METHODS}

The national surveillance system for BTV-8 in the Swiss cattle and sheep population was developed and evaluated in the following seven main steps: (1) The Swiss cattle and sheep population was stratified with regard to its risk of BTV-8 infection. (2) All relevant SSC for BTV-8 were identified and described. (3) The sensitivity of each SSC was quantified using stochastic scenario tree modelling. (4) The most influential parameters on the sensitivity of each SSC were identified and evaluated using sensitivity analysis. (5) Simple cost calculations were conducted for each SSC and compared in relation to the component sensitivities achieved. (6) The overall sensitivities of different combinations of SSC were calculated and the most cost-effective combination identified. And (7) The effectiveness of the prospective surveillance strategy was evaluated.

\subsection{Stratification of the population}

Because BT is a vector-borne disease, the main risk of incursion was expected in areas with vector presence. Infection via import of infected cattle and 
Table I. Categorization of Swiss cantons per geographical risk area for BTV-8 introduction in Switzerland for the summer months of 2006. The canton(s) listed in brackets $(<>)$ represent one grid cell.

\begin{tabular}{lcc}
\hline Low risk & \multicolumn{1}{c}{ Medium risk } & High-risk \\
\hline$<\mathrm{BE}$ south $>$ & $<\mathrm{AI}, \mathrm{AR}, \mathrm{SG}>$; $<\mathrm{BE}$ north $>$; $<\mathrm{BE}$ middle $>;$ & $<\mathrm{FR}>$; $<\mathrm{AG}>$; $<\mathrm{BS}, \mathrm{BL}, \mathrm{SO}>$; \\
& $<\mathrm{GR}>$; $<\mathrm{JU}, \mathrm{NE}>$; $<\mathrm{LU}>$; & $<\mathrm{GE}, \mathrm{VD}>$; $<\mathrm{TG}>$; $<\mathrm{TI}>$; $<\mathrm{ZH}, \mathrm{SH}>$ \\
& $<\mathrm{UR}, \mathrm{SZ}, \mathrm{OW}, \mathrm{NW}, \mathrm{GL}, \mathrm{ZG}>$; $<\mathrm{VS}>$ & \\
\hline
\end{tabular}

sheep was also considered initially. Yet due to the Swiss import regulations that came into effect in May 2007 (Technical Guidance 07/22, in force until 31st December 2007) where all ruminants and camels had to be tested for BT, this infection route was considered to be negligible and was therefore not included in the final model.

Temperature, altitude, and humidity are important factors which influence vector presence and activity. Therefore, vector habitats were determined through the creation of thematic maps for altitude, precipitation, and average temperatures [8] using data from 50 meteorological stations provided by the Swiss Meteorological Office for the year 2006. Maps of vector distribution were created and used in combination with cattle density maps in order to determine an approximation for the probability of a BTV-8 transmission from vector to cattle [9]. The final combined maps were then used to define different geographical risk areas with regard to the transmission of BTV-8 from vector to cattle. On the basis of EU recommendations ${ }^{3}$ for BT surveillance in Europe, Switzerland was divided into 16 grid cells taking into account both the administrative borders of cantons along with the expanse and the total number of susceptible cattle present. Cattle density was taken into account in order to categorize the 16 grid cells with regard to their geographical risk level. As an approximation, the same grid areas and geographical categorization were also used for sheep. Due to its relatively high cattle population, the canton of Bern was divided into three grids, namely Bern north, Bern middle, and Bern south. Several cantons with small areas and/or small cattle population were merged into one grid (Tab. I). Finally, each of the total 16 grid cells was attributed a geographical risk level $(3=$ high-risk;

\footnotetext{
${ }^{3}$ Commission regulation (EC) No. 1266/2007 of 26 October 2007 on implementing rules for Council Directive 2000/75/EC as regards the control, monitoring, surveillance, and restrictions on movements on certain animals of susceptible species in relation to Bluetongue.
}

$2=$ medium risk; $1=$ low risk) with regard to the different geographical risk areas of the final combined maps (Tab. I, Fig. 1).

\subsection{Identification of possible SSC}

In order to establish a cost-effective surveillance system for BTV-8 in Swiss cattle and sheep populations, possible active and passive SSC were identified and described.

\subsubsection{Active SSC}

Antibodies for BTV-8 can be detected in infected animals 7-10 days after infection using the serological competitive enzyme-linked immunosorbent assay (c-ELISA) test (VMRD, Inc., Pullman, WA, USA). Therefore, individual blood serology of cattle and sheep on farms (SEROFARM) and individual blood serology of cattle and sheep at slaughterhouse (SEROSLH) were identified to be two possible SSC for the detection of BTV-8 infected animals. The latter SSC was not considered further due to obviously high operational cost (see Sect. 3.2.). A further active SSC to detect BTV-8 infected dairy cattle was to conduct bulk milk tests (BMT) in dairy cattle herds using milk test ELISA (ID Screen ${ }^{\circledR}$ Blue Tongue Milk from ID Vet, Montpellier, France).

The sequence of steps leading to disease detection conducting SEROFARM and BMT, respectively, are depicted in Figures 2A and 2B.

\subsubsection{Passive SSC}

With regard to passive surveillance, the probability of detecting BTV-8 cases due to detection and reporting of infected animals with clinical signs through animal caretakers and veterinarians (CLIN) was evaluated. The crucial point in this process is the probability that the infected animals show clinical signs and that the measures leading to BT detection are taken by the farmers and veterinarians influenced 


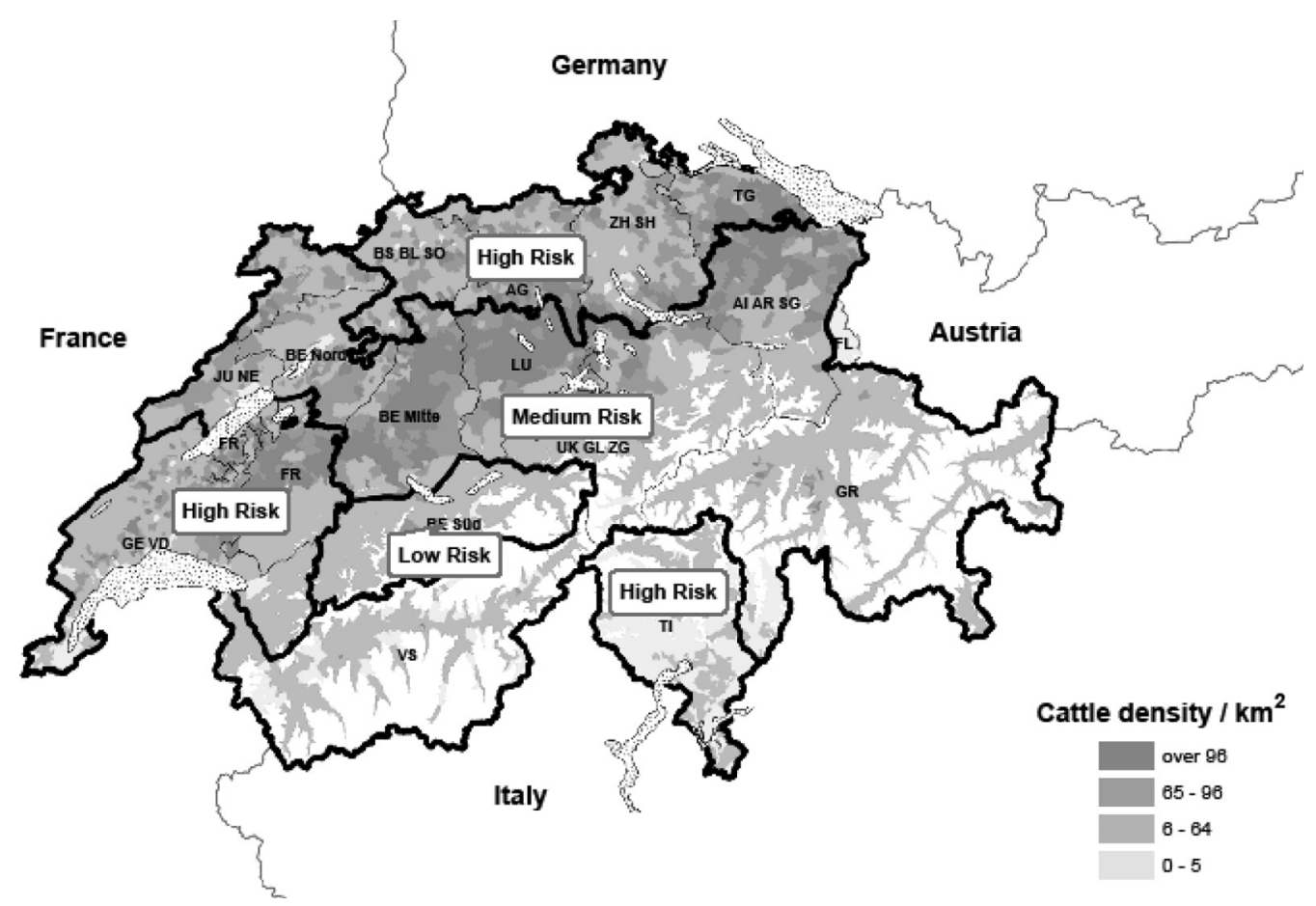

Figure 1. Map of Switzerland showing the cattle density and risk areas with cantonal distribution in Switzerland.
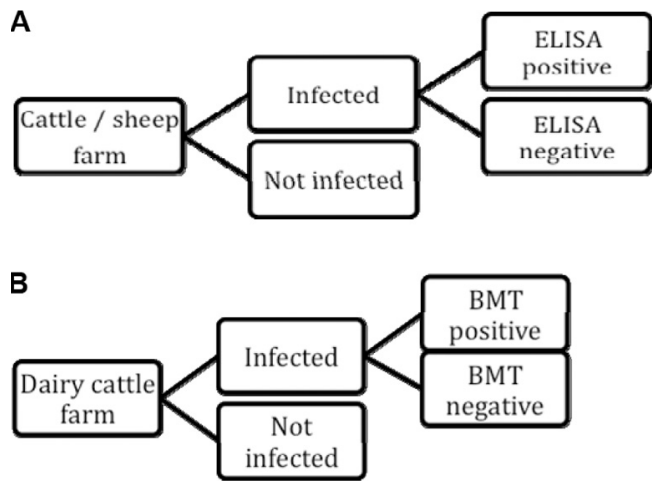

Figure 2. (A) Scenario tree for blood serology on cattle or sheep farm (SEROFARM) in Switzerland. (B) Scenario tree for bulk milk testing on dairy cattle farm (BMT) in Switzerland.

by their disease awareness (DA) levels (Fig. 3A). The probability of finding infected animals by serologically testing female cattle and sheep after an abortion (abortion testing, ABT) as a specific form of CLIN was a further evaluated passive SSC. In order to acquire a first estimate for the sensitivity of ABT, the scenario tree was kept as simple as possible (Fig. 3B). The probability of a farm having pregnant female animals aborting due to a BTV-8 infection was given by the probability for abortions in infected animals (Tab. II).

\subsection{Quantification of SSC sensitivities}

The sensitivity of each SSC (Component Sensitivity, CSe) was calculated according to Martin et al. [4] and Hadorn and Stärk [2], based on the scenario trees shown in Figures 2A, 2B, 3A, and 3B, respectively. Microsoft Excel and Palisade @RISK were used as the modelling software.

For all four evaluated SSC (SEROFARM, BMT, CLIN, and ABT), the probability of a cattle or sheep farm having BTV-8 infected animals is influenced by its geographical location, i.e. the geographical risk area and its corresponding risk level high, medium or low. The assumed proportions of cattle and sheep 
A

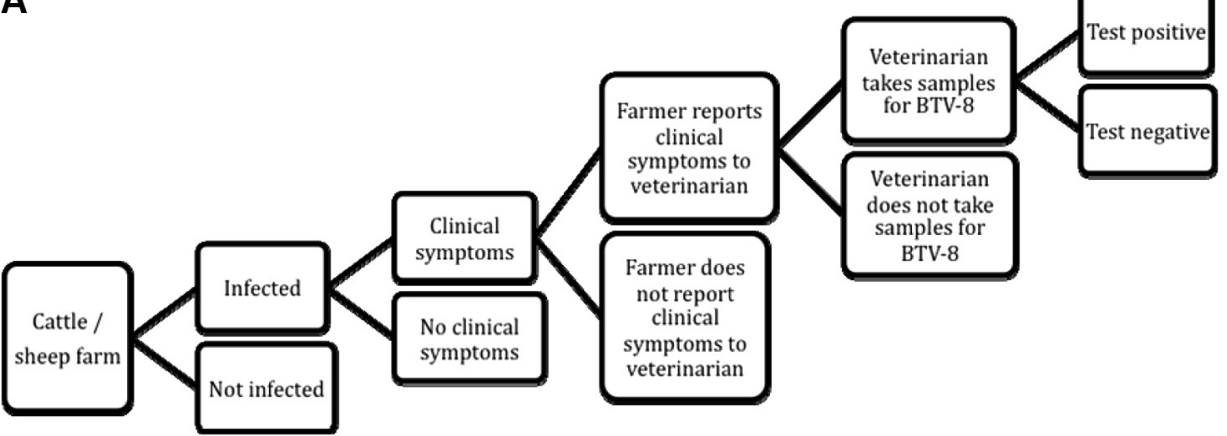

B

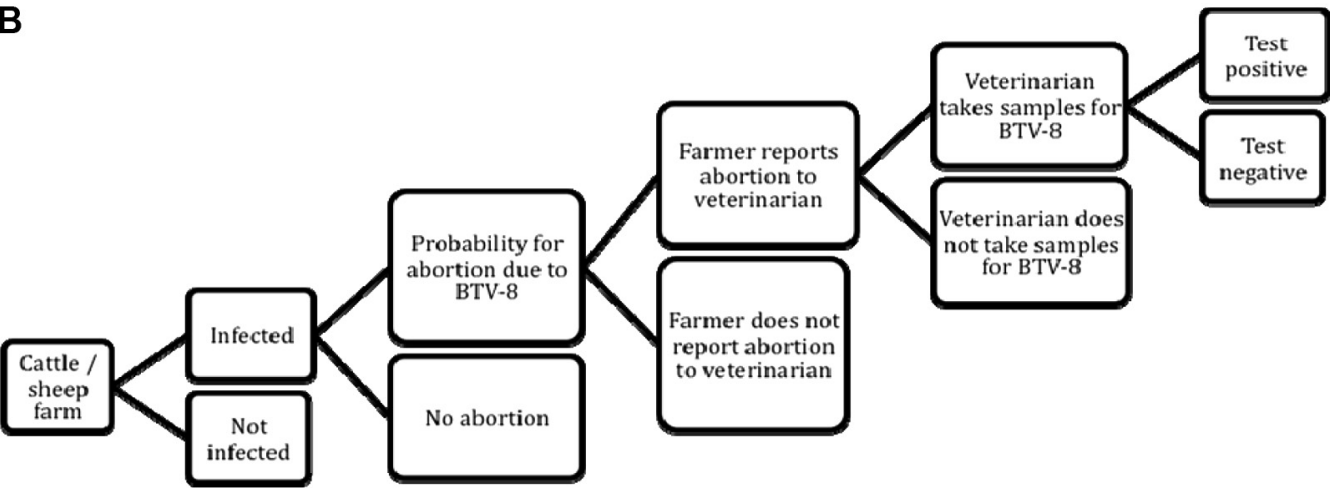

Figure 3. (A) Scenario tree for passive clinical surveillance on cattle or sheep farm (CLIN) in Switzerland. (B) Scenario tree for abortion testing on cattle or sheep farm (ABT) in Switzerland.

farms per geographical risk area are shown in Table III.

In order to incorporate the impact of the geographical risk levels on case occurrence, relative risks were implemented for the three different geographical risk areas [4]. Based on expert opinions from outbreaks in Belgium in 2006, it was assumed that the risk for infection was 25-times higher in high-risk areas and 10-times higher in medium risk areas, respectively, compared to low risk areas.

The farm-level design prevalence $(\mathrm{P} * \mathrm{H})$, i.e. the level of disease in the population to be detected by the SSC, was set to be $0.2 \%$ within the scope of this project. This means that a SSC is assessed regarding the probability that at least one infected farm would be detected if disease was present at a $\mathrm{P} * \mathrm{H}$ of $\geq 0.2 \%$. The test performances of the c-ELISA for SEROFARM and the milk test ELISA for BMT were assumed to be equal (Tab. II).

Due to the nature of the outbreak in 2006/2007 and the exclusive involvement of BTV-8, it was possible to collect clinical symptoms data from affected countries ${ }^{4}$ and use them as input parameters for CLIN and ABT (Tab. II). DA level of farmers and veterinarians, i.e. the probability of the farmer noticing clinical symptoms and contacting the veterinarian, and that of the veterinarian having a BT suspect and conducting the appropriate BT diagnostic test, were set arbitrarily according to personal experience and using four qualitative DA categories [2] described in Table IV. In spring 2007, the DA of cattle and sheep farmers was estimated to be low given the BTV-free status of Switzerland. At the time of working on the model, BTV was known to affect sheep more than cattle. Therefore, the probability that

\footnotetext{
${ }^{4}$ Elbers A.R.W., Mintiens K., Staubach C., Gerbier G., Meiswinkel R., Hendrickx G., et al., Bluetongue virus serotype 8 epidemic in North-western Europe in 2006: preliminary findings, Proceedings of the SVEPM conference, 2007, pp. 231-245.
} 
Table II. Input parameters used to model BTV-8 surveillance using stochastic scenario trees. The SSC considered are: SEROFARM = individual blood serology on farm; BMT = bulk milk testing on dairy cattle farm; CLIN = passive clinical surveillance in sheep and cattle farms, respectively; $A B T=$ abortion testing.

\begin{tabular}{|c|c|c|}
\hline $\begin{array}{l}\text { Description of input } \\
\text { parameter }\end{array}$ & Value & Source \\
\hline Design prevalence $\mathrm{P} * \mathrm{H}$ & 0.002 & Set arbitrarily \\
\hline $\begin{array}{l}\text { Sensitivity of blood } \\
\text { serology for SEROFARM }\end{array}$ & RiskPert $(0.99 ; 0.995 ; 1.00)$ & Personal opinion (based on data for c-ELISA) \\
\hline $\begin{array}{l}\text { Sensitivity of milk serology } \\
\text { for BMT }\end{array}$ & RiskPert $(0.99 ; 0.995 ; 1.00)$ & $\begin{array}{l}\text { Personal opinion (based on data for ID } \\
\qquad \text { Screen }^{\circledR} \text { ) }\end{array}$ \\
\hline $\begin{array}{l}\text { Probability for abortions } \\
\text { in cattle due to BTV-8 }\end{array}$ & RiskPert $(0.00 ; 0.016 ; 0.30)$ & [2] \\
\hline $\begin{array}{l}\text { Probability for clinical } \\
\text { symptoms in cattle } \\
\text { due to BTV-8 }\end{array}$ & RiskPert $(0.039 ; 0.07 ; 0.47)$ & [2] \\
\hline $\begin{array}{l}\text { Probability for clinical } \\
\text { symptoms in sheep due to BTV-8 }\end{array}$ & RiskPert $(0.047 ; 0.15 ; 0.48)$ & [2] \\
\hline $\begin{array}{l}\text { Probability that cattle } \\
\text { farmer calls veterinarian }\end{array}$ & RiskPert $(0.1 ; 0.2 ; 0.3)$ & Low estimated disease awareness \\
\hline $\begin{array}{l}\text { Probability that veterinarian } \\
\text { suspects BTV-infection in cattle }\end{array}$ & RiskPert $(0.1 ; 0.2 ; 0.3)$ & Low estimated disease awareness \\
\hline $\begin{array}{l}\text { Probability that sheep } \\
\text { farmer calls veterinarian }\end{array}$ & RiskPert $(0.1 ; 0.2 ; 0.3)$ & Low estimated disease awareness \\
\hline $\begin{array}{l}\text { Probability that veterinarian } \\
\text { suspects BTV-infection in sheep }\end{array}$ & RiskPert $(0.4 ; 0.5 ; 0.6)$ & Medium estimated disease awareness \\
\hline
\end{tabular}

Table III. Estimated proportion of cattle and sheep farms per geographical risk area for BTV-8 introduction in Switzerland for the summer months of 2006.

\begin{tabular}{lccc}
\hline & \multicolumn{3}{c}{$\begin{array}{c}\text { Proportion of farms per } \\
\text { geographical risk area }\end{array}$} \\
\cline { 2 - 4 } & Low risk & Medium risk & High-risk \\
\hline Cattle farms & $14.9 \%$ & $54.9 \%$ & $30.2 \%$ \\
Sheep farms & $55.5 \%$ & $18.0 \%$ & $26.5 \%$ \\
\hline
\end{tabular}

a veterinarian identified a $\mathrm{BT}$ suspect and took samples was set at medium in the sheep population compared to low in the cattle population (Tab. II).

Because the geographical risk area influences the probability of a herd being infected and therefore the probability of a positive test result, two different sampling designs were evaluated; Random sampling (RS) in all risk areas and targeted sampling (TS) in high-risk areas only. The latter was done as described by Hadorn and Stärk [2].
The number of processed units per SSC ( $n$ ) influences CSe, as it is shown in Equation 1:

$$
\mathrm{CSe}=1-(1-\mathrm{CSeU})^{n}
$$

where $\mathrm{CSeU}$ is the probability that any randomly chosen farm out of the population will give a positive outcome (for the equation how to calculate $\mathrm{CSeU}$, we refer to Martin et al. [4]). The number ${ }^{5}$ of processed units for CLIN in cattle and sheep is given with all cattle herds (52 983) and sheep flocks (14 116), respectively, in Switzerland. The number of processed units for SEROFARM and BMT was set to be 50,100, and 200 herds and flocks, respectively, and the number of abortions corresponding to the number of processed units for ABT was arbitrarily set to be 500 (Tab. V). Values for all input parameters were either used as fixed numbers or Pert distributions. The Pert distributions accounted for the uncertainty in the data, and permitted a range for minimum, most likely and maximum values to be used. All Monte Carlo simulations were run with 10000 iterations.

\footnotetext{
${ }_{5}$ Agrarinformationssystem Schweiz AGIS, 2005.
} 
Table IV. Disease awareness level categories used to model the probability of detecting clinical BTV-8 cases in sheep and cattle by farmers and veterinarians as part of a passive surveillance program in Switzerland.

\begin{tabular}{lccc}
\hline Category & \multicolumn{3}{c}{$\begin{array}{c}\text { Distribution parameters } \\
\text { (RiskPert } \text { distributions) }\end{array}$} \\
\cline { 2 - 4 } & $\begin{array}{c}\text { Minimum } \\
\text { value }\end{array}$ & $\begin{array}{c}\text { Most } \\
\text { likely } \\
\text { value }\end{array}$ & $\begin{array}{c}\text { Maximum } \\
\text { value }\end{array}$ \\
\hline $\begin{array}{l}\text { Low disease } \\
\text { awareness }\end{array}$ & 0.10 & 0.20 & 0.30 \\
$\begin{array}{l}\text { Medium disease } \\
\text { awareness }\end{array}$ & 0.40 & 0.50 & 0.60 \\
$\begin{array}{l}\text { High disease } \\
\text { awareness }\end{array}$ & 0.70 & 0.80 & 0.90 \\
$\begin{array}{l}\text { Very high disease } \\
\text { awareness }\end{array}$ & 1.00 & 1.00 & 1.00 \\
\hline
\end{tabular}

\subsection{Evaluation of influential parameters per SSC}

According to the sensitivity analysis of the stochastic scenario tree model, i.e. the regression coefficients of the@RISK output reports, the most influential parameters affecting each CSe could be identified. This information aided decision makers by identifying possible paths for improving the quality of the overall surveillance system.

\subsection{Cost calculations per SSC}

In order to decide on the most cost-effective SSC combination for the national surveillance system, cost in relation to the achieved CSe is crucial. Therefore, a simple calculation of cost was performed for each SSC. For SEROFARM, the following expenses per cattle farm were considered: the farm visit of the veterinarian, cost of blood-sampling, transport and subsequent diagnosis considering an average herd size of 30 cattle (Tab. VI). For SEROFARM in sheep, the same expenses were considered using an average flock size of 38 animals (Tab. VI).

For BMT, the only expenses per dairy cattle farm were the laboratory cost including aliquoting and postage of the bulk milk sample since the collection of milk samples could be integrated into the regular nationwide milk sampling procedure in terms of the Swiss quality program (Tab. VI). The costs of BMT were the same for each dairy cattle farm due to bulk milk testing.
For CLIN, the expenses generally include the cost of verification of suspects and possible expense for information campaigns in order to increase DA. The higher the DA becomes, the more suspect cases are reported, creating an increased number of diagnostic procedures and associated costs. Within the scope of this project, we considered the cost of 1 suspect cattle farm and 2 suspect sheep farms per month, given a low DA, and 3 suspect cattle farms and 5 suspect sheep farms given an increased DA (Tab. V). Further expenses for CLIN were calculated with a global fund of $50000 \mathrm{CHF}$ per year for an information campaign in order to increase DA in farmers and veterinarians (Tab. VI). For ABT, the expenses correspond to the number and cost of testing suspect cattle and sheep farms, respectively.

\subsection{Identification of an optimized surveillance system}

Within the scope of this project, it was assumed that all SSC of the surveillance system are independent. However, this may not be true for all SSC evaluated in this project. Therefore, it should be kept in mind that the overall sensitivity estimates may be slightly overestimated. The purpose of this approach was to explore relative sensitivities of several SSC combinations for priority setting and not primarily to obtain absolute sensitivity values. Hence, the overall surveillance system sensitivity (SSe) can be calculated using the following equation [4]:

$$
\mathrm{SSe}=1-\prod_{j=1}^{J}\left(1-\mathrm{CSe}_{j}\right)
$$

where $J$ is the number of SSC in the country and $\mathrm{CSe}_{j}$ is the SSC sensitivity of the $j$-th component. Using this equation, SSe through different combinations of SSC were calculated and evaluated in relation to the estimated cost in order to determine the most cost-effective strategy for the early detection of BTV-8 in Swiss cattle and sheep populations for 2007. It was assumed that managers would choose the combination of SSC which would provide a maximal cost-effectiveness.

\subsection{Quantification of SSC sensitivities on a monthly basis}

In order to estimate the effectiveness of the implemented surveillance system for BTV-8, CSe, and SSe were prospectively calculated for the year 2007 on a monthly basis. Because the distribution of the geographical risk areas per grid depends on the season, the proportion of farms per risk level were adapted 
Table V. Probability of detecting BTV-8 incursion in Switzerland at an assumed farm-level prevalence of 0.02 by using different SSC. SEROFARM = individual blood serology of cattle farm; BMT = bulk milk testing on dairy cattle farm; CLIN = passive clinical surveillance in sheep and cattle farm, respectively; ABT = abortion testing; $n=$ number of farms processed per SSC; RS = random sampling; TS = targeted sampling; DA level = disease awareness level; $\mathrm{L}=$ low disease awareness; $\mathrm{M}=$ medium disease awareness; $\mathrm{H}=$ high disease awareness; Vets = veterinarians; $\mathrm{CSe}=$ surveillance system component sensitivity; $\mathrm{SSe}=$ surveillance system sensitivity.

\begin{tabular}{|c|c|c|c|c|c|c|c|}
\hline \multirow[t]{2}{*}{ SSC } & \multirow[t]{2}{*}{ Survey design } & \multirow[t]{2}{*}{$n$} & \multirow[t]{2}{*}{ DA level } & \multicolumn{3}{|c|}{ Percentiles of CSe } & \multirow{2}{*}{$\begin{array}{c}\text { Costs per } \\
\text { month (CHF) } \\
\text { (based on Tab. VI) }\end{array}$} \\
\hline & & & & 5 th & 50th & 95th & \\
\hline \multirow[t]{4}{*}{ SEROFARM } & RS & 50 & - & 0.0945 & 0.0948 & 0.0951 & 52150 \\
\hline & RS & 100 & - & 0.1801 & 0.1806 & 0.1811 & 104300 \\
\hline & RS & 200 & - & 0.3278 & 0.3286 & 0.3294 & 208600 \\
\hline & TS & 200 & - & 0.5291 & 0.5302 & 0.5313 & 208600 \\
\hline \multirow[t]{4}{*}{ BMT } & RS & 50 & - & 0.0945 & 0.0948 & 0.0951 & 3100 \\
\hline & RS & 100 & - & 0.1801 & 0.1806 & 0.1811 & 6200 \\
\hline & RS & 200 & - & 0.3278 & 0.3286 & 0.3294 & 12400 \\
\hline & TS & 200 & - & 0.5291 & 0.5302 & 0.5313 & 12400 \\
\hline \multirow[t]{2}{*}{ CLIN cattle } & - & 52983 & L: farmer, vets & 0.1445 & 0.3813 & 0.7275 & $\begin{array}{l}1 \text { suspect cattle } \\
\text { case: } 1043\end{array}$ \\
\hline & - & 52983 & M: farmer, vets & 0.7114 & 0.9532 & 0.9992 & $\begin{array}{l}3 \text { suspect cattle } \\
\text { cases: } 3129\end{array}$ \\
\hline \multirow[t]{2}{*}{ CLIN sheep } & - & 14116 & L: farmer; M: vets & 0.1811 & 0.3830 & 0.6254 & $\begin{array}{l}2 \text { suspect sheep } \\
\text { cases: } 2614\end{array}$ \\
\hline & - & 14116 & M: farmer; H: vets & 0.5735 & 0.8624 & 0.9751 & $\begin{array}{l}5 \text { suspect sheep } \\
\text { cases: } 6535\end{array}$ \\
\hline ABT cattle & - & 500 & L: farmer, vets & $1.89 \mathrm{E}-04$ & $5.81 \mathrm{E}-04$ & $1.30 \mathrm{E}-03$ & $\begin{array}{l}500 \text { suspect cattle } \\
\text { farms: } 521500\end{array}$ \\
\hline $\begin{array}{l}\text { CLIN cattle and } \\
\text { CLIN sheep }\end{array}$ & - & & $\begin{array}{l}\text { L: cattle and sheep } \\
\text { farmer, vets in } \\
\text { CLIN cattle; M: vets } \\
\text { in CLIN sheep }\end{array}$ & 0.3973 & 0.6403 & 0.8586 & 3657 \\
\hline
\end{tabular}


Table V. Continued.

\begin{tabular}{|c|c|c|c|c|c|c|c|}
\hline \multirow[t]{2}{*}{$\begin{array}{l}\mathrm{SSC} \\
\end{array}$} & \multirow[t]{2}{*}{ Survey design } & \multirow[t]{2}{*}{$n$} & \multirow[t]{2}{*}{ DA level } & \multicolumn{3}{|c|}{ Percentiles of CSe } & \multirow{2}{*}{$\begin{array}{c}\text { Costs per } \\
\text { month (CHF) } \\
\text { (based on Tab. VI) }\end{array}$} \\
\hline & & & & 5 th & 50 th & 95 th & \\
\hline $\begin{array}{l}\text { CLIN cattle and } \\
\text { CLIN sheep }\end{array}$ & - & & $\begin{array}{l}\text { L: cattle farmer, vets } \\
\text { in CLIN cattle; M: sheep } \\
\text { farmer; H: vets in } \\
\text { CLIN sheep }\end{array}$ & 0.7249 & 0.9237 & 0.9877 & $\begin{array}{c}7578 \\
\text { (without expenses } \\
\text { for information } \\
\text { campaigns in order } \\
\text { to increase DA) }\end{array}$ \\
\hline $\begin{array}{l}\text { BMT and CLIN } \\
\text { cattle and } \\
\text { CLIN sheep }\end{array}$ & $\mathrm{RS}$ & 200 & $\begin{array}{c}\text { L: cattle and sheep } \\
\text { farmer, vets in CLIN } \\
\text { cattle; M: vets in CLIN } \\
\text { sheep }\end{array}$ & 0.5954 & 0.7585 & 0.9051 & 16057 \\
\hline $\begin{array}{l}\text { BMT and CLIN } \\
\text { cattle and } \\
\text { CLIN sheep }\end{array}$ & TS & 200 & $\begin{array}{c}\text { L: cattle and } \\
\text { sheep farmer, vets in } \\
\text { CLIN cattle; M: } \\
\text { vets in CLIN sheep }\end{array}$ & 0.7169 & 0.8310 & 0.9336 & 16057 \\
\hline $\begin{array}{l}\text { BMT and CLIN } \\
\text { cattle and } \\
\text { CLIN sheep }\end{array}$ & TS & 200 & $\begin{array}{c}\text { L: cattle farmer, } \\
\text { vets in CLIN cattle; } \\
\text { M: sheep farmer; H: vets in } \\
\text { CLIN sheep }\end{array}$ & 0.8707 & 0.9642 & 0.9942 & $\begin{array}{l}19977 \\
\text { (without expenses } \\
\text { for information } \\
\text { campaigns in order } \\
\text { to increase DA) }\end{array}$ \\
\hline
\end{tabular}


Table VI. Input values per processed unit for estimating the cost of different SSC being considered for detection of BTV-8 incursions in Switzerland.

\begin{tabular}{|c|c|c|c|}
\hline SSC & Processed unit & Matter of expense & Cost (CHF) \\
\hline \multirow{5}{*}{$\begin{array}{l}\text { Individual blood } \\
\text { serology of cattle } \\
\text { (SEROFARM cattle) }\end{array}$} & $\begin{array}{l}\text { Cattle farm } \\
\text { (average herd size }\end{array}$ & $\begin{array}{l}\text { Farm visit cost } \\
\text { (veterinarian) }\end{array}$ & 28 \\
\hline & estimated at 30 animals) & Blood-sampling per cattle & $30 * 12$ \\
\hline & & $\begin{array}{l}\text { Transport of samples } \\
\text { to laboratory }\end{array}$ & 25 \\
\hline & & $\begin{array}{l}\text { Cost of diagnostic } \\
\text { per sample }\end{array}$ & $30 * 21$ \\
\hline & & Total cost per cattle farm & 1043 \\
\hline \multirow{5}{*}{$\begin{array}{l}\text { Individual blood } \\
\text { serology of sheep } \\
\text { (SEROFARM sheep) }\end{array}$} & Sheep farm & Farm visit cost (veterinarian) & 28 \\
\hline & $\begin{array}{l}\text { (average herd size } \\
\text { estimated at } 38 \text { animals) }\end{array}$ & Blood-sampling per sheep & $38 * 12$ \\
\hline & & $\begin{array}{l}\text { Transport of samples } \\
\text { to laboratory }\end{array}$ & 25 \\
\hline & & $\begin{array}{l}\text { Cost of diagnostic } \\
\text { per sample }\end{array}$ & $38 * 21$ \\
\hline & & Total cost per sheep farm & 1307 \\
\hline \multirow[t]{2}{*}{$\begin{array}{l}\text { Bulk milk testing } \\
\text { on dairy cattle } \\
\text { farm (BMT) }\end{array}$} & Dairy cattle farm & $\begin{array}{c}\text { Laboratory cost } \\
\text { including aliquoting and } \\
\text { postage }\end{array}$ & 62 \\
\hline & & Total cost per dairy cattle farm & 62 \\
\hline \multirow[t]{3}{*}{$\begin{array}{l}\text { Passive clinical } \\
\text { surveillance (CLIN) }\end{array}$} & $\begin{array}{c}\text { Cost in order to increase disease } \\
\text { awareness level }\end{array}$ & Assumed cost per year & 50000 \\
\hline & $\begin{array}{c}\text { Cost of testing one average suspect } \\
\text { cattle farm given a low disease } \\
\text { awareness level }\end{array}$ & $\begin{array}{l}\text { Cost of clarification } \\
\text { of one assumed } \\
\text { suspect cattle farm }\end{array}$ & 1043 \\
\hline & $\begin{array}{l}\text { Cost of testing two average suspect sheep } \\
\text { farms given a low disease awareness level }\end{array}$ & $\begin{array}{l}\text { Cost of clarification } \\
\text { of two assumed } \\
\text { suspect sheep farms }\end{array}$ & 2614 \\
\hline
\end{tabular}

monthly with regard to the average climatic factors based on meteorological data of the year 2006 (data not shown but available on request from the corresponding author). Not only did we take the seasonal factor into account for the overall assessment of the implemented surveillance system, but we also changed the values of DA of people involved taking into account planned and already conducted information campaigns concerning $\mathrm{BT}$, knowledge of the pattern of the vector season and reports on the changes in the BTV-8 situation in Northern Europe that might influence DA of farmers and veterinarians. Consequently, for CLIN in cattle a low DA in farmers and veterinarians was assigned to the months of January to June, and a low-medium DA from July to December. For CLIN in sheep, low DA in farmers and veterinarians was used for the months of January to July, a low-medium DA level was used for August and September, ending with a medium-high DA level for October to December. This medium-high level of DA was used to reflect the planned information campaigns for sheep farmers and veterinarians in order to improve CLIN in sheep.

\section{RESULTS}

\subsection{Stratification of the population}

The cattle density with the distribution of the 26 Swiss cantons per grid and the categorization of the cantons per geographical risk area is 
shown in Figure 1. The corresponding estimated proportions of cattle and sheep farms per geographical risk level are shown in Table III.

\subsection{Assessment of possible SSC}

In Switzerland, slaughterhouse collection of specimens is not very common yet. Therefore, the operational costs of serological sampling and tracing back of animals would be relatively high. Together with a lower sensitivity of the c-ELISA if used in single animals compared to the use as a herd test, SEROSLH did not provide advantages compared to SEROFARM and was not considered further as an economical alternative for the national surveillance system. SEROFARM is feasible for cattle herds as well as for sheep flocks. But because this SSC requires the visit of a veterinarian per farm and each animal has to be bled separately, the high amount of work and cost was a clear disadvantage. Bleeding of sheep is still more laborious than bleeding of cattle without providing advantages with regard to CSe. Therefore, SEROFARM was only kept in the analysis with regard to cattle.

CLIN is an important SSC because it is ongoing and has comprehensive coverage of the population. Therefore, CLIN was also analyzed further for both cattle and sheep population. In order to cover the sheep population sufficiently with regard to BT surveillance, a specific SSC replacing SEROFARM had to be considered. At the time of developing this surveillance system, characteristic clinical signs for BTV-8 in sheep were anticipated. Therefore, it was planned to generally enhance passive CLIN in sheep by conducting specific information campaigns and courses for sheep farmers and practitioners. Additionally, a selected number of sheep farmers in high-risk areas should be trained. Those farmers should examine their sheep regularly with the idea of doing active CLIN.

In Switzerland, a regular nationwide milk sampling procedure already exists in terms of a quality program. The monthly collected bulk milk samples for the quality program can therefore also be used for BMT in dairy cattle with the advantage that logistical efforts can be optimized. The testing of all female cattle and sheep having aborted (ABT) seemed to be a feasible option and was therefore also assessed using stochastic scenario tree modelling.

\subsection{Quantification of SSC sensitivities}

In order to evaluate SEROFARM in cattle, simulations were conducted for a sample size of 50, 100, and 200 cattle farms. SEROFARM provided a CSe of $9.5 \%$ and $18.1 \%$ for a sample size of 50 and 100 cattle farms, respectively. Conducting a blood serological survey in 200 randomly chosen cattle herds per month (random sampling, RS), a SEROFARM-CSe of 32.9\% could be achieved (Tab. V). If 200 cattle herds in geographical high-risk areas were tested (targeted sampling, TS), the achieved SEROFARM-CSe could be increased to up to $53.0 \%$ (Tab. V). The BMT-CSe with a sample size of 50,100 , and 200 dairy cattle herds, respectively, were the same as for SEROFARM due to the equal detection process (Fig. 2B) and the same estimated test sensitivity; namely $9.5 \%, 18.1 \%$, and $32.9 \%$ for RS in 50,100 , and 200 cattle herds, respectively, and $53.0 \%$ for TS in 200 dairy cattle herds. The CLIN-CSe in cattle for a population size of 52983 herds was calculated with an estimated low DA in farmers and veterinarians and provided a CSe of $38.1 \%$. CLINCSe for a sheep population of 14116 flocks was calculated to be $38.3 \%$ estimating a low DA in farmers and a medium DA in veterinarians. Although the number of processed farms is almost 3.8-times lower in cattle than in sheep, CLIN-CSe in sheep was almost equal to CLIN$\mathrm{CSe}$ in cattle. This supported the strategy of enhancing passive CLIN and establishing active CLIN in sheep population as an alternative for SEROFARM in sheep. ABT in cattle produced a negligible CSe of 5.8E-04\% assuming the testing of aborted fetal material from 500 animals from 500 different farms. Therefore, this SSC did not contribute sufficiently to the national surveillance system, and the idea was abandoned to test all female animals having aborted for BTV-8.

\subsection{Evaluation of influential parameters per SSC}

For SEROFARM and BMT, the only influencing parameter was the sensitivity of the tests 
used. Therefore, the only parameter that can be influenced in order to increase $\mathrm{CSe}$ was the choice of the tests. In CLIN, the most influential parameter was the probability of infected animals showing clinical signs. This parameter is given by the infectious agent and cannot be influenced by management. But the DA of farmers and veterinarians was also shown to be an influential parameter for CLIN. Therefore, CLIN-CSe can potentially be increased by conducting information campaigns to enhance the DA in farmers and veterinarians. At the time of establishing the Swiss BT surveillance system, BT was known to be a disease occurring especially in sheep population causing typical clinical symptoms. Therefore, decision makers decided to specifically intensify CLIN in sheep instead of conducting serological sampling in this population.

\subsection{Cost calculations per SSC}

The cost calculations were done for each SSC per processed unit and then added to the total cost per month. It is evident that SEROFARM in cattle with an estimated cost of $1043 \mathrm{CHF}$ per herd would clearly be more costly than BMT with $62 \mathrm{CHF}$ per dairy cattle farm (Tab. VI): the total average expenses for SEROFARM with a sample size of 200 cattle herds per month amounted to $208600 \mathrm{CHF}$ compared to $12400 \mathrm{CHF}$ per month for BMT in 200 dairy cattle herds (Tab. V). The average total monthly cost of CLIN in cattle and sheep were estimated with $3657 \mathrm{CHF}$ considering one suspect cattle herd and two suspect sheep flocks for verification (Tab. V).

\subsection{Identification of an optimized surveillance system}

The combination of CLIN in cattle and sheep assuming a low to medium DA provided a SSe of $64.0 \%$ with expenses assumed to be $3657 \mathrm{CHF}$ (Tab. V). Analyzing the model for an increased DA, the SSe could be enhanced to $92.4 \%$ with assumed cost of 7578 CHF (Tab. V), without taking into account the cost of conducting DA campaigns. The cost of a surveillance system composed of CLIN in cattle and sheep and
BMT in 200 randomly chosen dairy cattle farms (BMT_RS) added up to $16057 \mathrm{CHF}$ providing a SSe of $75.9 \%$ (Tab. V). The cost of CLIN with BMT in 200 dairy cattle farms situated in highrisk areas (BMT_TS) was the same with $16057 \mathrm{CHF}$ but providing a higher SSe of $83.1 \%$. Simulating the model with the increased DA data in sheep farmers (medium DA) and veterinarians dealing with suspect sheep cases (high DA) and combining this with BMT_TS and CLIN cattle, the SSe could be increased up to $96.4 \%$ with the assumed monthly cost of 19 977 CHF (Tab. V).

\subsection{Quantification of SSC sensitivities on a monthly basis}

The sensitivities of the three SSC of the prospective surveillance system, i.e. BMT_TS, CLIN cattle and CLIN sheep, were simulated on a monthly basis and the overall SSe was determined per month (Fig. 4). For BMT_TS, small increases in CSe were expected in the months of July and October, although it was expected to remain on a relatively constant rate of $30-40 \%$ with a slight decrease in the winter months. Prior to July, this CSe was at $0 \%$ due to the fact that testing was planned to start in July 2007. The CLIN-CSe for cattle predicted one main peak increase seen in late July due to the estimated increase in DA related to the release and distribution of a BT documentary to stakeholders, and the raised number of reports in Swiss newspapers. The estimated average sensitivity for this SSC ranged from 35\% (pre-awareness campaign) to $80 \%$ after release of the BT documentary. CLIN in sheep predicted two main increases, whereby the first increase being due to the activity mentioned above, along with a second increase in DA brought by the education program destined for a selected number of sheep farmers. The overall SSe provided a sensitivity level ranging from $45 \%$ early on in the year to circa $96 \%$ when all activities were implemented.

\section{DISCUSSION}

In this work, scenario tree modelling was used as a tool in order to plan and implement 


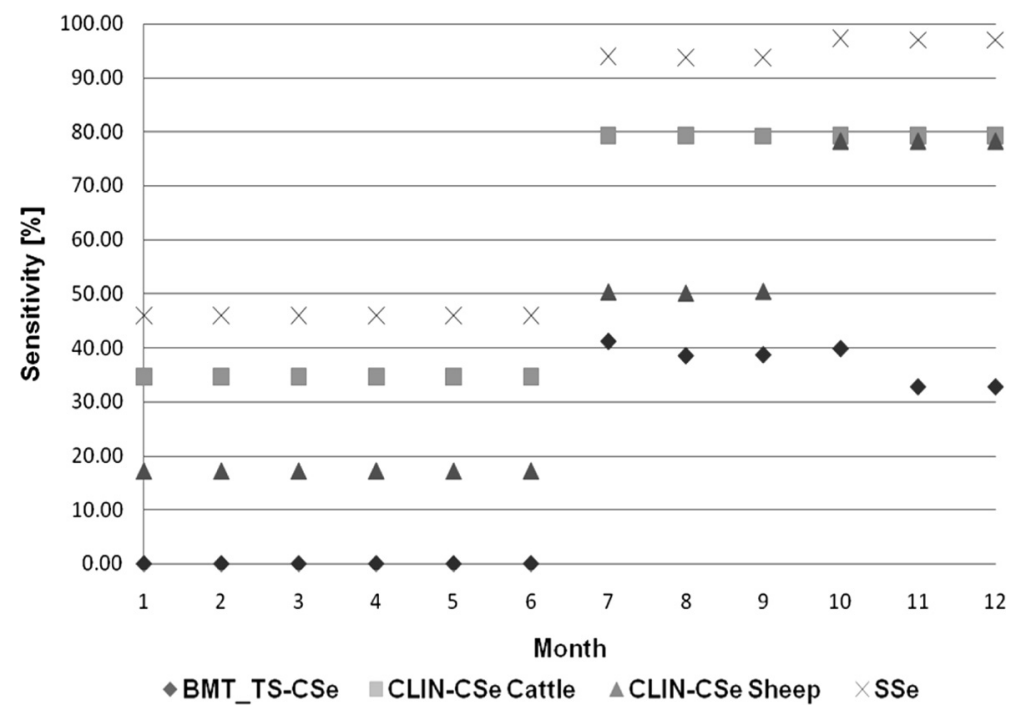

Figure 4. Median sensitivity of the bulk milk testing program in 200 dairy cattle herds in high-risk areas (BMT TS-CSe), of clinical surveillance in cattle (CLIN-CSe Cattle) and sheep (CLIN-CSe Sheep) and of the median overall surveillance system sensitivity (SSe) of detecting BTV-8 cases (sensitivity, \%) in Switzerland over a period of one year. The sensitivity calculations were done per month and the numbers refer to the respective month $(1=$ January, $2=$ February, $3=$ March etc. $)$.

a cost-effective national surveillance system. It was shown that the structured assessment of different possible SSC offered the possibility to identify strengths and weaknesses of surveillance activities in a timely fashion and also helped to identify approaches where changes are likely to be most cost-effective. Because resources are generally decreasing, a rigorous evaluation of surveillance activities before their implementation rather than retrospectively is important in order to assure optimal use of finances and personnel.

The results of this scenario tree modelling enabled Swiss policy makers to decide on the optimal national surveillance system concerning BTV-8 based on the analysis of various surveillance-system options. Additionally, by identifying the most influential parameters of the selected SSC with regard to the provided CSe, special actions could be taken to strengthen these parts of the surveillance system. As a further input of the scenario tree model, cattle and sheep farms were stratified according to geographical risk and, as a consequence, BMT was conducted as a targeted surveillance taking into account dairy cattle farms located in high-risk areas.

The results in Table $\mathrm{V}$ show that there was no statistically significant difference between scenarios. The results were communicated to the authorities who considered them in their decision on how to implement the surveillance system for BTV-8 in Switzerland. It was decided to base the surveillance system on the combination of BMT in 200 dairy cattle herds located in high-risk areas in addition to CLIN in cattle and a specifically increased CLIN in sheep. Despite the absence of a statistically significant change, there was an important improvement in SSe from $64 \%$ to $96 \%(50 \%$ values, Tab. V) for a relatively minor cost of around CHF 20000 per month. This investment was considered justified by the authorities. Identifying DA in farmers and veterinarians to be an important influential parameter on CLIN-CSe, it was decided to start an information campaign in order to increase DA and to 
enhance CLIN-CSe and consequently the overall SSe. Consequently, a DVD about BT was produced in order to increase DA in farmers and veterinarians. Additionally, workshops on BT have been organized in different regions of the country in order to increase DA levels, especially in the sheep industry. Direct results of the enhanced DA could be seen in the increasing amount of clinical suspect cases being reported after the launch of the various information campaigns. At the beginning of 2007, the DA for BT was assumed to be not very high and only 3 suspect cases for BT were reported in the first 5 months of the year. But after the specific information campaigns in June 2007 , the number of suspect cases increased to 24 in the next 4 months. After the first BT case was detected in Switzerland in October 2007, a further increase in the reporting of suspect cases was recorded (61 suspect cases in November 2007). The evaluation of the national surveillance system for BVT-8 on a monthly basis predicted that CLIN in cattle provided the highest CSe followed by CLIN-CSe in sheep and BMT_TS-CSe. Therefore, it was expected to detect the first BTV-8 case in Switzerland clinically on a cattle farm located in a high-risk area. Effectively, the first BTV-8 case in Switzerland was detected clinically in a cow on 28th of October 2007 in the canton of Basel City, categorized as high-risk area. The second case was detected via BMT_TS, and the 3rd to 5 th case were also detected clinically in cattle. Therefore, the occurrence of BTV-8 outbreaks in Switzerland provided a unique field validation of assumptions and results of this method in terms of CSe, the importance of DA and importance of the identified risk areas.

The output of stochastic scenario tree models will only be as good as the quality of the input parameters. Although some input parameters were uncertain due to lack of data, the relative contribution of different SSC to the planned national surveillance system and the influence of specific input parameters are considered to be sufficiently robust to be used as decision support.

The approach described in this paper is applicable also in other countries and to other epidemic diseases. Therefore, it has the potential to become a standard tool for the design of new surveillance systems for emerging diseases.

Acknowledgements. We would like to thank Christian Griot and Barbara Thür, Institute of Virology and Immunoprophylaxis, Mittelhäusern, Switzerland, as well as Eric Breidenbach, Martin Reist, Hansueli Ochs and Lukas Perler, Swiss Federal Veterinary Office, for their inputs. We would also like to thank Michael Binggeli, Swiss Federal Veterinary Office, for designing the map in Figure 1.

\section{REFERENCES}

[1] Doherr M.G., Audigé L., Monitoring and surveillance for rare health-related events: a review from the veterinary perspective, Philos. Trans. R. Soc. Lond. B Biol. Sci. (2001) 356:1097-1106.

[2] Hadorn D.C., Stärk K.D.C., Evaluation and optimization of surveillance systems for rare and emerging infectious diseases, Vet. Res. (2008) 39:57.

[3] Lilienfeld D.E., Stolley P.D., Foundations of epidemiology, 3rd ed., Oxford University Press, Oxford, UK, 1994.

[4] Martin P.A., Cameron A.R., Greiner M., Demonstrating freedom from disease using multiple complex data sources 1: a new methodology based on scenario trees, Prev. Vet. Med. (2007) 79:71-97.

[5] Mehlhorn H., Walldorf V., Klimpel S., Jahn B., Jaeger F., Eschweiler J., et al., First occurrence of Culicoides obsoletus-transmitted Bluetongue virus epidemic in Central Europe, Parasitol. Res. (2007) 101:219-228.

[6] Meiswinkel R., Baldet T., de Deken R., Takken W., Delécolle J.-C., Mellor P.S., The 2006 outbreak of Bluetongue in northern Europe - The entomological perspective, Prev. Vet. Med. (2008) 87:55-63.

[7] Purse B.V., Mellor P.S., Rogers D.J., Samuel A.R., Mertens P.P., Baylis M., Climate change and the recent emergence of Bluetongue in Europe, Nat. Rev. Microbiol. (2005) 3:171-181.

[8] Racloz V., Presi P., Vounatsou P., Schwermer H., Casati S., Vanzetti T., et al., Use of mapping and statistical modelling for the prediction of Bluetongue occurrence in Switzerland based on vector biology, Vet. Ital. (2007) 43:513-519.

[9] Racloz V., Venter G., Griot C., Stärk K.D.C., Estimating the temporal and spatial risk of Bluetongue related to the incursion of infected vectors into Switzerland, BMC Vet. Res. (2008) 4:42. 